\title{
PLANAR PROJECTION OF MOBILE LASER SCANNING DATA IN TUNNELS
}

\author{
J. A. Gonçalves ${ }^{1}$, Mendes, R. ${ }^{1}$, Araújo $^{1}$, E., Oliveira, A. ${ }^{2}$, Boavida, J. ${ }^{2}$ \\ ${ }^{1}$ Universidade do Porto - Faculdade de Ciências, Rua Campo Alegre, 687, 4169-007, Porto, Portugal - \\ jagoncal@fc.up.pt, rlpmendes@gmail.com, emilia.s.araujo@gmail.com \\ 2 Artescan - 3D Scanning, IPN Incubadora de Empresas, 3030-199 Coimbra, Portugal - \\ (adrianooliveira,jboavida)@artescan.net
}

KEY WORDS: Laser scanning, point cloud, automation, adjustment, rectification

\begin{abstract}
:
Laser scanning is now a common technology in the surveying and monitoring of large engineering infrastructures, such as tunnels, both in motorways and railways. Extended possibilities exist now with the mobile terrestrial laser scanning systems, which produce very large data sets that need efficient processing techniques in order to facilitate their exploitation and usability.

This paper deals with the implementation of a methodology for processing and presenting 3D point clouds acquired by laser scanning in tunnels, making use of the approximately cylindrical shape of tunnels. There is a need for a $2 \mathrm{D}$ presentation of the 3D point clouds, in order to facilitate the inspection of important features as well as to easily obtain their spatial location.

An algorithm was developed to treat automatically point clouds obtained in tunnels in order to produce rectified images that can be analysed.

Tests were carried with data acquired with static and mobile Riegl laser scanning systems, by Artescan company, in highway tunnels in Portugal and Spain, with very satisfactory results. The final planar image is an alternative way of data presentation where image analysis tools can be used to analyze the laser intensity in order to detect problems in the tunnel structure.
\end{abstract}

\section{INTRODUCTION}

Laser scanning systems are an important technology in the monitoring of engineering infrastructures. The very large data sets of point clouds provide a detailed geometric description of the observed objects, which is useful to monitor the conditions of the structure (Yoon, et al., 2009). For several years laser scanning was static, with equipment mounted on a tripod. In recent years mobile systems, integrating very accurate positioning and navigation (INS-GNSS) became operational and a commercial solution in last decade (Hunter, 2009). Fast laser surveys along roads and railways became possible, allowing for accurate monitoring of infrastructure conditions. Automation in data analysis has also developed, allowing for faster results (Biosca and Lerma, 2008)

Most of the systems now used incorporate digital cameras, synchronized with the laser, in a way that the point cloud can be coloured in order to create a realistic model of the objects. The image component provides important information, for example to detect possible damages in structures.

The intensity of the laser pulse returns can also be treated in the same way as image data. It may be of particular interest in structures not illuminated, such as tunnels, where the detection of cracks, moisture, water intrusions may be detectable in the laser intensity data (Nuttens et al., 2010).

In the case of laser intensity or any imagery from other sensors, optical or thermal, the data volumes are very large and visual inspection may not be sufficient to detect problems on the surface of tunnels being monitored. Standard image processing techniques can be used for an automatic detection but it requires that surfaces are transformed onto planes in order that the data can be represented as a planar image. This is a problem of transforming a surface into a plane, similar to a map projection. Our problem is then the identification of the main surface in the point cloud, decide which points are not on the surface, and then define it mathematically. In a rough tunnel it is likely that points may be slightly separated from the surface, being described by the distance to this reference surface, in the same way as a height. Once the surface is defined it is then transformed in some manner into a plan. Points are described by their $(x, y)$ coordinates on the plan, and possibly a distance to the reference surface, and can be mapped uniquely onto the original $3 \mathrm{D}$ position.

A simple tunnel with the shape of a cylinder is the simplest problem. It can be unfolded, transforming it in a plane, without any deformation. The shape of the cylinder cross-section is not a problem, provided that it is always the same. The resulting plane has two coordinates that are the distance along the cylinder axis and the distance along the cross-section.

Real tunnels differ from this situation in that they are curved and can be modelled as juxtaposed sections of a torus, in a snake shape. Figure 1 shows a torus and two sections of a torus juxtaposed.
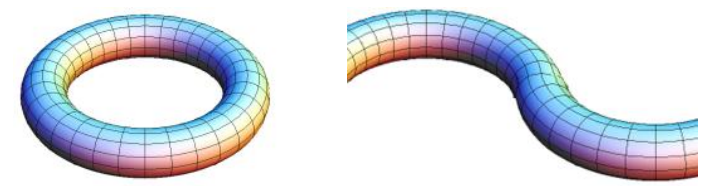

Figure 1. Representation of a torus and the juxtaposition of two torus sections.

This paper describes an algorithm that takes a point cloud and identifies the tunnel alignment, fits curves to the cross-sections profiles and divides the tunnel in small sections, mapping it then into a plane. The curvature of tunnels, especially in railways, is small, normally with curvature radius of several kilometres. The deformation that will occur is very small, not degrading the 
perception of dimensions in the rectified image of the tunnel surface.

This method was developed in cooperation with Artescan-3D Scanning, a Portuguese company that owns a Riegl VMX-250CS6 mobile laser scanning system (figure 2). This system has been applied in long tunnel surveying, both motorway and railway (Boavida et al., 2012). These can be as-built surveys of tunnels, or can be made with the purpose of monitoring and maintenance. Many of these surveys span along distances of several kilometres, producing very large amounts of data. The availability of methodologies that automatically produce rectified, planar images of the tunnels, was found an essential tool for analysis of these data.

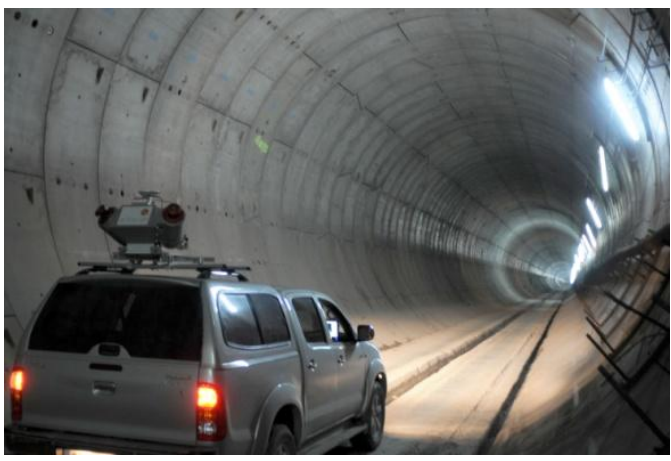

Figure 2. Riegl VMX-250-CS6 mobile laser scanning system in operation in a tunnel survey.

The method was tested with data of railway tunnels, first in small samples of the underground of the city of Porto, and then with data collected in the survey of a $25 \mathrm{~km}$ long high-speed railway tunnel (Pajares tunnel in Asturias, Spain). Figure 3 shows a profile of the terrain and the tunnel in a distance of more than $1 \mathrm{~km}$. Tunnel slope is approximately $1.7 \%$.

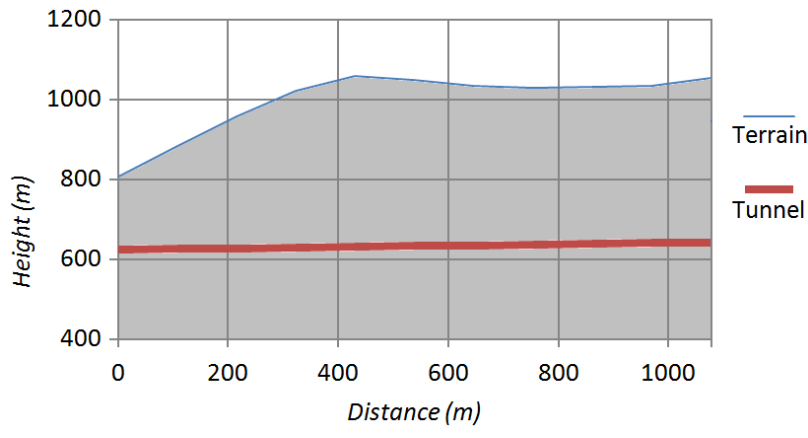

Figure 3. Profile of the terrain and the tunnel in Asturias.

\section{DESCRIPTION OF THE ALGORITHM}

The goal of this algorithm is to project a 3D tunnel surface into a plan, or in other words cut the tunnel longitudinally and unfold it to make it flat. The input is a point cloud acquired by laser scanning inside a tunnel.

The algorithm was programmed in PostgreSQL/PostGIS, making use of some of its capabilities of spatial search and analysis.

The algorithm is composed by the following 5 stages.

\section{STAGE 1}

The first stage is to recalculate the tunnel axis. The original axis can be acquired in different ways. It can be extracted from the vehicle trajectory (obtained by the inertial navigation system); from a traverse or other survey network; from a manual procedure or from a semi automatic process involving a skeletonization algorithm. Once a planar representation of the axis is available, a set of points, with constant spacing, will be generated along the axis (figure 4). This spacing (e.g. 10 meters) is a parameter defined by the user.

\section{$\stackrel{0}{\longmapsto} \quad \perp^{10} \quad 20$ Meters}

Figure 4. Tunnel axis with the original points (larger squares) and the equally spaced points (small dots)

For each one of these points a line is created, locally perpendicular to the axis. The direction of the axis in a point is considered to be the direction defined by the previous point and the next point, as shown in Figure 5.

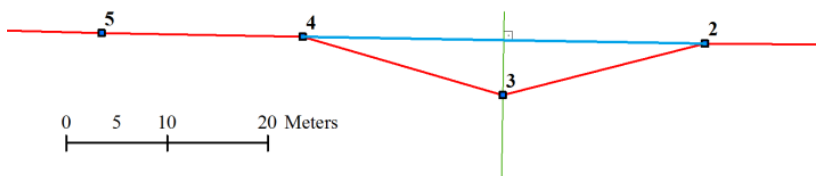

Figure 5. The perpendicular line (green line) at point 3 is calculated perpendicularly to the direction defined (blue line) by the previous (2) and the next point (4).

Now a buffer around this perpendicular line is created, with parameters specified by the user. Then, all the points from the point cloud that are inside this buffer are averaged to calculate the central point of the tunnel at the respective location (the central point is a $3 \mathrm{D}$ point calculated with the average values of the three coordinates of the selected points from the point cloud). The final 3D tunnel axis is composed by all this $3 \mathrm{D}$ points. This process can be recursively repeated until the differences between the old and the new axis are negligible.

\section{STAGE 2}

The second stage is to rectify the tunnel axis that was automatically created. It is possible that the axis calculated in the previous stage has errors or unwanted points. In these cases, it is crucial to examine the result and make the desired changes manually, before continuing to the next stage. This step is essentially a visual inspection and possibly a manual adjustment of few points. It is not time consuming and may increase the results quality in irregular parts of the tunnel.

\section{STAGE 3}

The third stage is the segmentation of the point cloud. For each vertex of the axis a plan perpendicular to the trajectory is calculated (direction defined as before, between the previous point and the next point). These plans can be materialized with their dimension defined by user (Figure 6).

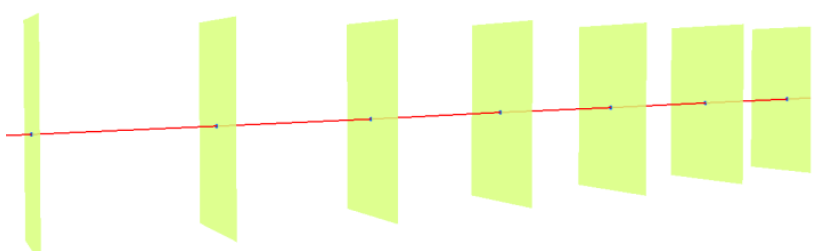

Figure 6 . The tunnel axis (red line) is cut by perpendicular plans (green plans) crossing the axis in its vertices, segmenting it.

The tunnel axis is cut in $n-1$ segments ( $n$ is the number of axis vertices). For each segment, comprehended between two 
plans, all points from the point cloud in the segment are identified and tagged with the corresponding segment identifier (Figure 7).

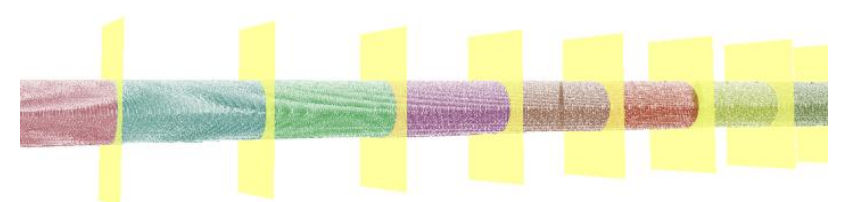

Figure 7. Point cloud classified by axis segment (limited by yellow plans).

\section{STAGE 4}

The fourth stage is to create two sections for each axis segment. For each axis segment a point is fixed at $25 \%$ of the segment length from the first point (called the main point), and a plan perpendicular to the axis (called the main plan) is calculated. The same is done for a point at $75 \%$ of the segment, as shown in figure 8:

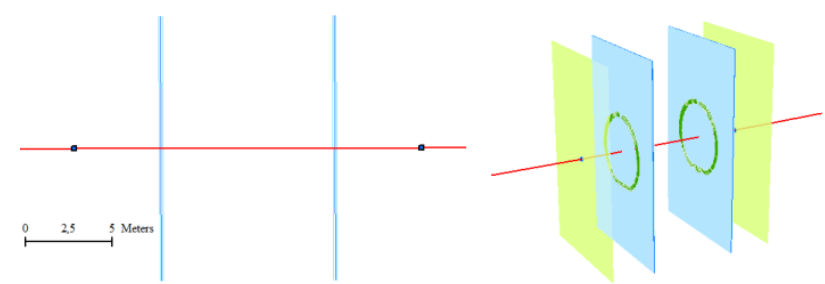

Figure 8 . The tunnel axis is cut at $25 \%$ and $75 \%$ (blue plans).

The aim of these two intermediate plans is to obtain the cross section of the tunnel. Each of these plans is used to define a box, with a thickness of 1 or 2 decimetres along the axis. Now, all the points from the point cloud, inside this box are extracted, projected in this plan and their polar coordinates (radius and angle) are calculated. The angle is calculated with origin at the upward direction (not exactly the vertical because of the axis slope). The selected points are grouped by angular classes, with a spacing defined by the user. For each angular interval the average and standard deviation of the radius are calculated, and those that differ from the mean more than two standard deviations, are excluded from the selection (only at this stage). With the others the centroid of the point set is calculated, and this mean point will be considered a point of the cross-section. The cross-section is composed by all the centroids of the angular intervals (Figure 9). This stage is repeated to calculate the second section of the tunnel segment, at $75 \%$ of the distance. At the final, two sections per segment are obtained.

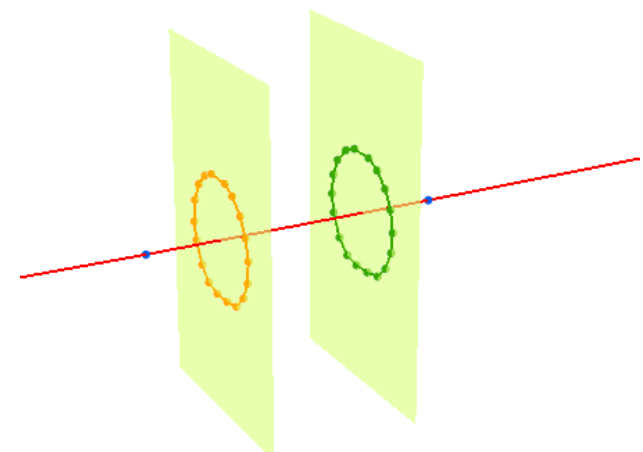

Figure 9. The two sections (at $25 \%$ (green) and $75 \%$ (orange) of the segment length) and the respective points.

\section{STAGE 5}

The fifth stage is to project the points of the point cloud to a plan. For all the points of the point cloud the nearest crosssection is determined (the $25 \%$ or the $75 \%$ one). Then, the two nearest points of the cross-section are obtained, as well as the corresponding points on the other section, as shown in Figure 10 .

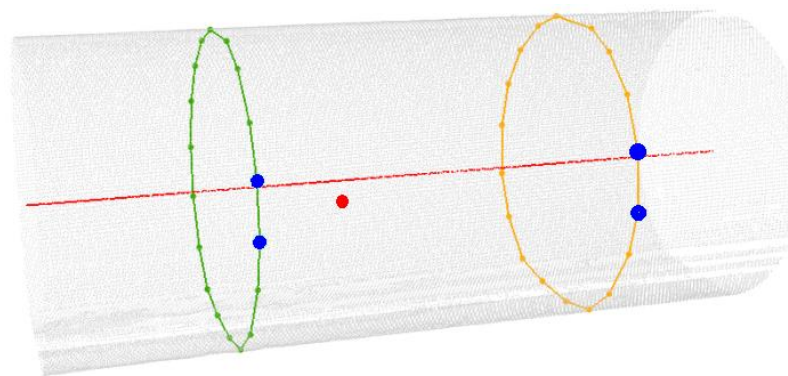

Figure 10. The two closest points in the cross-section and its homologous in the other section (4 blue points) for the point under analysis (red point).

The top of the tunnel is the origin for the determination of the distance along the cross-section. The point in analysis is projected under the line between the two points of the section and his length along the wall (along the section) until the top of the tunnel is measured (this step is repeated for the other section as well). Now, the distance between the point in analysis and the top of the tunnel is calculated through the weighted average of the two lengths, calculated before along the sections, assuming the weights are the distances between the point and the two sections (Figure 11).

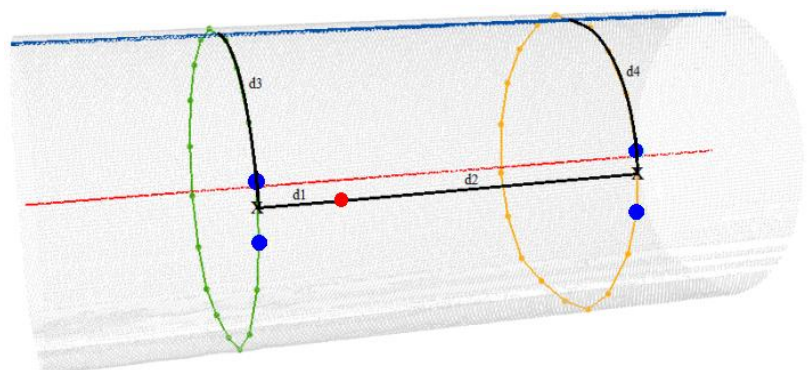

Figure 11. The distance between the point in analysis and the top of the tunnel (blue line) along the two cross-sections (black arcs), calculated by a weighted average of the two distances.

In Figure 11 the distance from the point in analysis (red point) to the top of the tunnel (blue line) is calculated with the average of the distances along the sections.

$$
\left\{\begin{array}{l}
X=X_{0}+\left(\frac{d_{1} d_{3}+d_{2} d_{4}}{d_{1}+d_{2}}\right) \sin (\theta) \\
Y=Y_{0}+\left(\frac{d_{1} d_{3}+d_{2} d_{4}}{d_{1}+d_{2}}\right) \cos (\theta)
\end{array}\right.
$$

where $\quad d_{1}, d_{2}=$ distance between point and cross-sections $d_{3}, d_{4}=$ length of the arcs along the cross-sections $\theta=$ axis segment's azimuth $X_{0}, Y_{0}=$ coord. of the point intersection at the axis

All the points in the point cloud that are at a distance larger than a predefined tolerance (defined by the user) are considered not to be on the tunnel surface (eg. cables) and discarded from the projection process. Finally, the resulting points (2D points) have $\mathrm{X}$ coordinate as the distance along the tunnel axis and the $\mathrm{Y}$ 
coordinate as the length of the arc (along the tunnel's wall), from the top of the tunnel to the point. The points from the point cloud are now projected in a plane. Laser return intensity values can then be resampled in order to generate a continuous image. Some examples are shown in the following section.

\section{RESULTS AND DISCUSSION}

This section describes some tests of the algorithm with real data. The initial tests were done with small subsamples of data collected in Portugal. A second test was done with the full data set acquired in Spain, but in subsamples of $1 \mathrm{~km}$. The choice of several parameters is justified.

\subsection{Tests with small samples}

The generation of an axis for a tunnel is a relatively simple process, by skeletonization, that can be achieved fully automatically. The definition of perpendicular plans in order to divide the tunnel in segments (in general with $10 \mathrm{~m}$ length) is also a simple and automatic process.

Once points in a segment are extracted there is an important parameter to chose, that depends on the shape of the crosssection. The first example is for a tunnel in the region of Porto, that has a rather irregular shape.

Figure 12(a) represents the points in a cross section, for which polar coordinates are calculated. In order to create a set of points that define the polyline of the cross-section, points are grouped in angle intervals, such as $5^{\circ}, 10^{\circ}$ or $20^{\circ}$. This corresponds to the cross-section being modelled by 72,36 or 18 points, respectively. Figure 12(b) represents, in green, blue and red these situations.

(a)

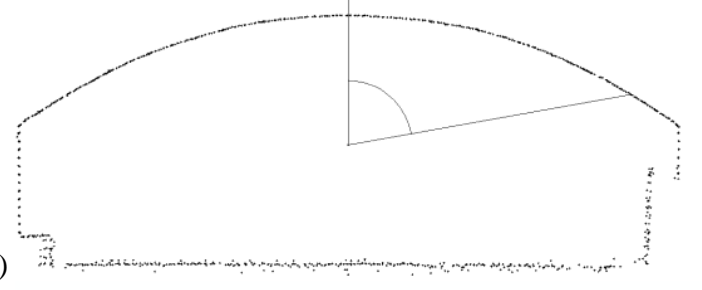

(b)

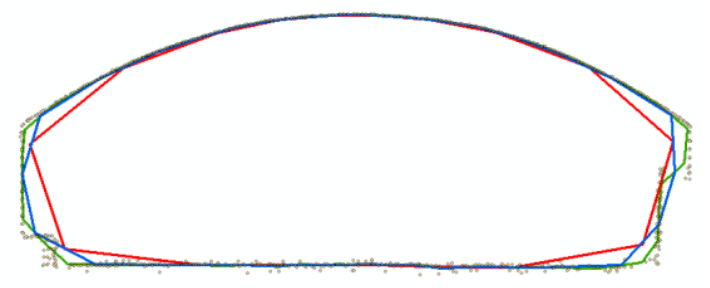

Figure 12. Points of a cross-section, in polar coordinates (a); adjustment of polylines at angular steps of $5^{\circ}, 10^{\circ}$ and $20^{\circ}$

At the smoother part, the top of the tunnel, an angle step of $20^{\circ}$ was enough. However, since in some parts the orientation of the line changes significantly, the adjusted line lies a few decimetres apart form the points. A $5^{\circ}$ step was chosen in order to do a better modelling of the cross-section.

Another important step is the elimination of points that do not belong to the tunnel surface, such as cables. The rule that was adopted was that, once a polyline was adjusted to the crosssection, any point that is more than half meter away from the polyline is not on the tunnel surface. Figure 13 shows a few points in cables. This step is important in order to avoid obstacles in the final unfolded image of the tunnel surface.

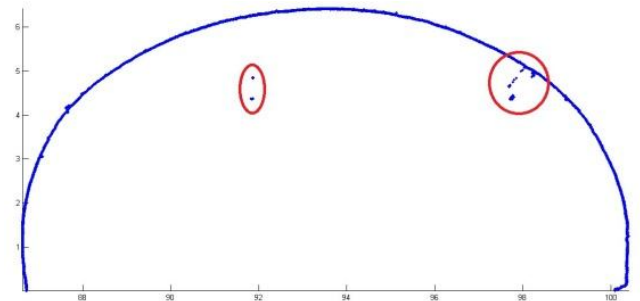

Figure 13. Elimination of points not on the surface.

Finally comes the planar projection. All points in the point cloud identified as belonging to the tunnel surface were mapped to a tunnel segment. Within the segment each point was located by a distance along the axis, and by a distance along the crosssection, in order to plot the segment as a planar image.

Figure 14 shows a segment of the tunnel in Porto, with grey values representing intensity. After unfolded it shown as a planar image of laser intensity, with the railways in the centre. The left and right parts of the image represent the top of the tunnel.
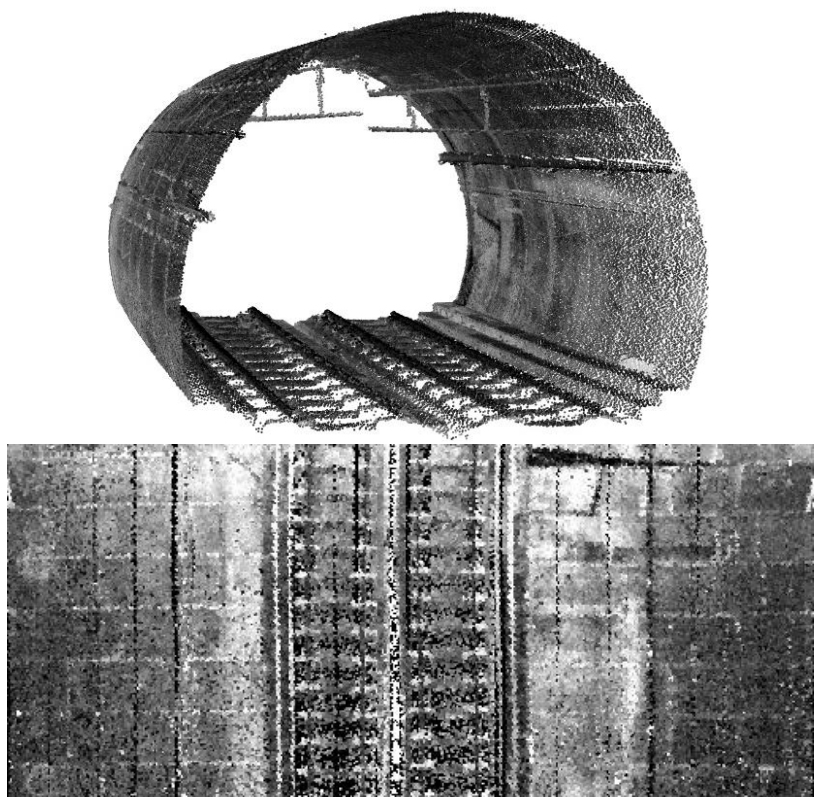

Figure 14. Unfolding of a tunnel segment

\subsection{Tests with larger datasets}

The Pajares tunnel, in Spain, with $25 \mathrm{~km}$ length, and a point spacing of a few mm, corresponds to an extremely large dataset that has to be treated partially. It was divided in $1 \mathrm{~km}$ parts and also these were decimated in the Riegl software in order to keep points in an average density of 1 point per square decimetre. This was enough for the definition of the surface, but all the data can be later processed for the planar images.

A few important differences existed in this case. First, the radius of curvature of this tunnel is very large (more than $4 \mathrm{~km}$ ), and so the tunnel segments could be increased to more than 10 meters without any problems with deformations.

Another fact for this tunnel is that its cross-section is nearly circular, with the bottom flat. A total of 36 points per crosssection are enough to have an approximation of $1.5 \mathrm{~cm}$ in a tunnel with $8 \mathrm{~m}$ diameter.

Although the tunnel surface is very regular in some parts it has some derivations that may cause some irregularities in the automatic determination of the tunnel axis. These very few situations were corrected by a manual editing, in a few points only, of the axis. 


\section{SUMMARY AND CONCLUSIONS}

An algorithm for automatic treatment of point clouds obtained by mobile laser scanning in tunnels was described. It identifies the tunnel axis, defines perpendicular cross-sections and maps all points on the tunnel surface into a coordinate system based on distance along the axis and distance along the section. This allows for a projection of the tunnel onto a planar surface, and generate an image of laser return intensities (or imagery of any other sensor). Any point of this planar image can be mapped back into 3D object space.

This resulting image can be analyzed, both visually or by means of image processing techniques, in order to detect anomalies in the tunnel, such as fractures or water infiltrations. This can be an important tool for a maintenance program, in order to reduce data analysis time.

The process is essentially automatic and is planned to be incorporated in software created by Artescan to provide non specialist users with essential automatic analysis tools, such as this one.

\section{Acknowledgments}

This project was support by program COMPETE, of the "Quadro de Referência Estratégico Nacional", financed by the European Regional Development Fund.

\section{References from Journals:}

Biosca J., Lerma J.L., 2008, Unsupervised robust planar segmentation of terrestrial laser scanner point clouds based on fuzzy clustering methods. ISPRS Journal of Photogrammetry and Remote Sensing. 63 (2008), pp. 84-98.

Yoon, J., Sagong, M., Lee, J. S., Lee, K., 2009. Feature extraction of a concrete tunnel liner from 3D laser scanning data. NDT \& E International 42(2), pp. 97-105.

\section{References from Other Literature:}

Boavida, J., Oliveira, A., Santos, B., 2012. Precise long tunnel survey using the Riegl VMX-250 Mobile Laser Scanning System. Riegl LiDAR 2012, Orlando, Florida, USA, Feb 27 Mar 01, 2012.

Hunter, G., 2009. Mobile Mapping - The Street Mapper Approach. Photogrammetric Week 2009. pp 179-190.

Nuttens,T., De Wulf, A., Bral, L., De Wit, B., Carlier, L, De Ryck, M., Constales, D., De Backer, H., 2010. High Resolution Terrestrial Laser Scanning for Tunnel Deformation Measurements. FIG Congress 2010, Australia, 11-16 April 2010. 